\section{From Preventative To Predictive Maintenance: The Organisational Challenge}

\author{
Luminita Ciocoiu, Carys E. Siemieniuch and Ella-Mae Hubbard ${ }^{1}$
}

Journal Title $X X(X): 1-$

11

QThe Author(s) 2016

Reprints and permission:

sagepub.co.uk/journalsPermissions.nav

DOI: $10.1177 /$ ToBeAssigned

www.sagepub.com/

(\$) SAGE

\begin{abstract}
Introduction of new technology (technology implementation) within an organisation can have wide reaching implications, beyond the effectiveness and efficiency savings that are typically the aim of such an endeavour. The 'Health and Prognostic Assessment of Railway Assets for Predictive Maintenance' (HPA) project developed a prognostic tool, which aimed to support enhancement of London Underground's Remote Condition Monitoring (RCM) system to support change from reactive and preventative to predictive maintenance, in order to increase effectiveness and efficiency and reduce LCH (Lost Customer Hours). This paper investigates the organisational challenges associated with the introduction of such a tool. The paper describes the approach adopted to model the extant maintenance processes (focusing on role mapping) and associated organisational structures which revealed issues such as unclear processes, poor communication and data sharing links and problems with delineation of responsibility for decision making. It also describes the development of a new maintenance process model that incorporates the additional functionality of the new prognostic tool, taking in to account changes of roles, responsibilities, organisational processes and activities.
\end{abstract}

\title{
Keywords
}

Maintenance; Remote Condition Monitoring; Organisational Change

\section{Introduction}

Maintaining a suitable level of performance is necessary over the lifecycle of a system and asset due to the importance of ensuring ongoing availability. ${ }^{1}$. Beyond a minimum performance level, in today's environment there is a growing demand for optimising the situation by maximising asset availability and safety, maintaining the operational quality and customer satisfaction, all whilst minimising costs ${ }^{2}$. As a result, the importance of an efficacious maintenance strategy has seen an upsurge across many industries. This supports a focus on optimisation of resource allocation and use, as this is where a competitive advantage can be gained. ${ }^{3}$.

Health Management Systems (HMS) can provide information about ongoing asset health during system oper- ation, which can support organisations to achieve better resource allocation within their maintenance activities and even develop the capability to act before failure occurs. These business needs are reflected in the drive for change from reactive focused maintenance practices to proactive maintenance practices.

When it comes to proactive actions, there are typically two main approaches: scheduled maintenance based on asset failure history and scheduled maintenance based on asset condition monitoring. Monitoring of the asset condition can be done either at location/ on-site or remotely, the later being the type of maintenance action (based on remote condition monitoring) that this paper is concerned with.

\section{Remote Condition Monitoring (RCM) systems}

RCM systems are innovative e-maintenance solutions that exploit emerging and developing technological advancements. Employing such systems can allow an organisation to implement a key paradigm change in the way maintenance is conducted. ${ }^{4}$.Traditional maintenance relies on the physical proximity of the maintenance team to the asset to enable them to observe status to achieve both monitoring of the asset condition and maintenance intervention. It is not possible or practical to physically inspect some assets. This may be

\footnotetext{
${ }^{1}$ Wolfson School of Mechanical, Electrical and Manufacturing Engineering, Loughborough University, UK

Corresponding author:

Ella-Mae Hubbard, Engineering Systems of Systems Research Group, Loughborough University, LE11 3TU, UK.

Email: E.Hubbard@lboro.ac.uk
} 
due to access issues, health and safety concerns or because of the cost of undertaking such activity. This in turn means that nascent defects may lay dormant, undetected until they cause a wider system failure. In order to eliminate some of the issues of physical inspection, many organisations are employing RCM systems, allowing a remote alternative for condition monitoring. RCM systems collect data from instrumented assets and display that data at a different location. A variety of RCM systems are in widespread use, supporting the provision of maintenance related services, such as diagnostics and quality assurance ${ }^{5}$.

The functional aim of a RCM system is to automatically and continuously monitor various aspects of the health of an asset during operation, with no need to halt or interrupt the operation for inspection. The behaviour should be checked against standard or expected behaviour to allow identification of any deviation from normal performance. The nature of this information then enables it to be made available on demand, to various decision makers, thus offering them decision support independent of time, location or organisational position $^{6}$.

The use of RCM systems allows organisations to exploit capabilities not previously available, allowing new insight into asset behaviour and performance during operation. Such systems can, and should, lead to new practices and rules ${ }^{4}$ in order to best implement the positive potential outputs of such systems, enabling proactive maintenance actions to be taken in advance of failure, and in a timely fashion to minimise disruption to operational performance.

London Underground hope to make use of such systems, with all the benefits they can bring, and to support this, the HPA project and consortium was created. Its purpose was to develop a prognostic tool to enhance existing RCM capability within London Underground and further to support the shift in maintenance practices necessary to successfully establish a shift from reactive and preventative towards predictive maintenance (with a proactive focus).

Passenger journeys on the London Underground network are on the increase, and expected to top half a million a day within the next five years. It has never been more important, nor more of a challenge, to keep the overall system in a safe and efficient operational mode. Any required maintenance can cause disruption for passengers, which is in turn costly for the organisation (beyond the basic cost for the required works). Being able to plan for when and where to deploy maintenance resources (without having to be reactive) to maximum effect is critical in minimising disruption and controlling costs.

\section{The Prognostic Tool}

The HPA project aimed to develop a prognostic tool which could be integrated into the existing London Underground RCM system in order to enhance the capability of the organisation to monitor the condition of various systems and assets and, in turn, to support decision-makers by providing actionable (but advisory) information regarding the Remaining Useful Life (RUL) of the assets. Such an enhancement should enable the organisation to be more intelligent and strategic with how it manages asset degradation and plans maintenance interventions ${ }^{7}$. An initial focus on escalators provided a sample case, with opportunity investigated to apply the tool to other fixed infrastructure and rolling stock assets.

The prognostic tool developed is a software-based tool that calculates RUL based on 'live' asset condition data and failure trends. The main functions of the tool are:

- To analyse the condition indicators (CIs) from the existing RCM database,

- To detect events in the data,

- To calculate RUL,

- To indicate optimum intervention time

A top level view of the Prognostic Tool role within the maintenance process is depicted in Figure 1, with data and information used and produced.

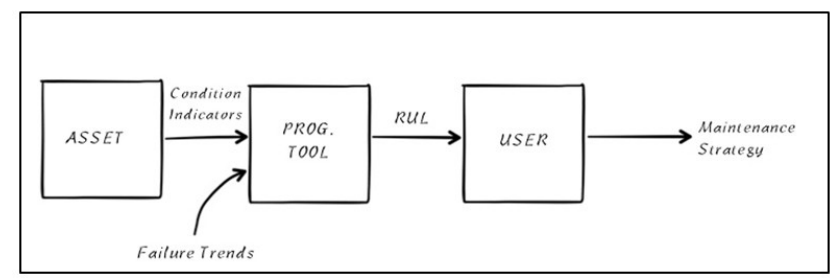

Figure 1. Top level process

It is worth noting that since the asset and system monitoring takes place during operation, the tool outputs are dynamic. This means that the outputs (including RUL information) will change over time as the condition of the asset changes. Also, the data upon which the analysis is based is gathered from sensors placed on the asset. It details physical symptoms but provides no insight into what is causing such symptoms (reflecting effect rather than cause). This is one of the reasons that the prognostic tool is defined and intended for use as a decision support tool, rather than a decision making tool. The decision making authority remains with the relevant decision makers. Identifying the difference between decision making and decision support is important as it influences the interaction with users (primary, secondary 
and tertiary), how they perceive the tool and the actions they take.

It is clear that advances in this area can have a positive impact on maintenance activities, along with important benefits for the overall organisation. However, getting the technology right is not the end of the story. For successful implementation, there are other questions to answer, for example:

- Will users trust the technology and the data it produces?

- Is the organisation ready for and capable of implementing any necessary changes in structure, processes, responsibilities?

- Have any training needs been identified? etc.

These questions affect the organisational and human context. Not getting these things right may not only mean that the technology is not successfully implemented, but could even impact on the wider operation of the organisation.

According to the literature ${ }^{8 ; 9}$, in spite of the significant

benefits that a prognostic capability could provide an organisation, many of the projects that seek to implement such a change fail to achieve their objective.

\section{Organisational challenges}

Introducing new technology ('technology implementation') has wider reaching, sometimes subtle and hidden, implications for organisational processes than the immediate benefits it may have been designed for. Within the context of moving to e-maintenance, research regarding organisational impact is in fact limited, but does identify ${ }^{4 ; 9}$ challenging areas (or areas of concern) that appear to be consistent, for example:

- The significance of shared ontology;

- Effective knowledge sharing, especially for relevant decision makers;

- Clearly defined and articulated roles and responsibilities for all those effected by the change;

- Clarity and transparency of the organisational strategy.

This list is not exhaustive, but findings such as these highlight the importance of key consideration and careful design of the organisational changes necessary if the new system, such as the HPA prognostic tool, is going to be implemented successfully and meet its designed targets. Such considerations should include, but not necessarily be limited to, comprehensive models of current and future processes, roles and information flows. Indeed, ignoring such challenges can lead to failure of the target implementation.
The cost of investigating such organisational challenge must, necessarily, be considered alongside other commitments when making any cost/ benefit decisions for the new implementation. The challenges should not be ignored. If their consideration pushes the costs too high, it is better to understand that early, rather than face costly interventions later in the project, or a complete failure.

Significantly, focus must not simply be on the new context following technology implementation, or indeed any key change within an organisation. The initial step should be to understand and detail the previous/ existing organisational culture, context and work practices, enabling the change to be implemented within a well mapped organisation - it is imperative you know where you have come from if you want any change to be truly successful.

One area to be conscious of is culture. In Hodgson et al ${ }^{10}$, culture is defined as "....an unconsciously acquired, shared set of values, preferences, attitudes, beliefs and rules that influence individual and group emotions and their behaviours towards individuals, the environment and other artefacts." Culture is seen ${ }^{10 ; 11}$ as being impacted at three key levels: ethnic, professional and organisational. In business, organisational cultures reflect the business leaders, markets, customers, products etc. they can be changed, butsuccessful change requires prolonged effort and 'organisational pain'. Furthermore, conflicts between the three levels of culture can also create divergent views and understanding through different work practices, hierarchies and expectations, particularly along a supply chain consisting of different types and sizes of organisation. An additional source of cultural differences can come from within teams operating within and between companies. Team effectiveness can be seriously impaired if large cultural diversity is found within a team.

Traditional maintenance practices typically rely on physical evidence to assess and interpret the condition of an asset and on people's skills and experience in interpreting the observable symptoms. Such experience will influence the way people work, allowing them to adapt and optimise their activity. These aspects are typically not clearly captured in formal processes, if they are considered at all (formal processes usually capture how a process/ activity 'should be' carried out, rather than how it is carried out). Therefore, when considering any change within an organisation or working environment, the introduction of a new process, system, technology or any innovation, it is important

to understand the way people work and the motivation/ rationale behind any existing or potential adaptive behaviour.

The condition based assessment approach, based exclusively on on-site monitoring, triggers three key challenges 
to address when considering and implementing a change towards RCM:

- Loss of tacit knowledge;

- The need for those involved in the whole process to have trust in non-physically observable data and any interpretations of it;

- Dependence on the reliability and ease of use of the technology.

Within the maintenance domain, workers will develop their own predictions based on experience and familiarity with the asset and its behaviour, based on patterns within the physical and observable cues, although this may not be conscious and is not likely to be captured within any formal process. The predictive nature of the prognostic tool (based on ongoing data collected from a variety of sensors) means that patterns may be identified long before

a maintenance engineer could identify from physically observable behaviour from the asset alone. For example, the prognostic tool may detect a significant variation in the performance data of a gear box, before the gear box shows any visible signs of failure. Without this physically observable behaviour to confirm deterioration, engineers have to rely on information provided by the RCM system. In order to be able to take timely and appropriate decisions,

they have to understand and trust that information. The outputs from the prognostic tool provide information about the condition to allow better planning. It does not, and should not, trigger action without further contextual considerations.

At the other end of the trust in technology spectrum however, it can be said that people can become too dependent on the technology and overlook its limitations. Jonsson and colleagues ${ }^{4}$ reported that "with more online measurements the less people will be walking around by the machines [...] In this case the remote diagnostic system led to a local physical distance as the value of a local presence was not realised. To the operator, the phone call from the remote technician had become a work practice that would indicate all problems with the machine and, therefore, the walk rounds were neglected. This shows that through the use of remote diagnostic systems follows the critical issue to manage the boundaries between the remote and local maintenance work." (p.215).

Wherever any level of automation or autonomy is introduced, the rationale and implications must be considered. Automation in systems often occurs for 'negative' reasons (e.g. the availability of more advanced technology or in reaction to accidents) or as a result of the 'Left-Over' principle ${ }^{12 ; 13}$ (whereby the designer will automate anything that can be automated and leave the remainder for the human operator(s). Unfortunately, these approaches to automation take into account neither the value added by human operator(s) nor the implications of interactions between the technical and non-technical elements of the system in question. Autonomy, on the other hand, has more to do with the locus of control and decision-making within a system or subsystem. In a manufacturing context, Sheridan ${ }^{14}$ describes autonomy on a 10-point scale, moving from 'The computer offers no assistance, human must decide all' to 'The computer decides everything and acts autonomously, ignoring the human'. PACT (Pilot Authority and Control of Tasks) also describes layers of autonomy to define clear operational relationships between the pilot and the UAV.Full autonomy, in the sense described by Sheridan ${ }^{14}$ is unlikely in the foreseeable future, and hence it is reasonable to suggest that humans will continue to be involved in the decision-making loop - Sheridan ${ }^{15}$ captures the intrinsic paradox of human and machine capability.

The purpose of RCM systems is to continually monitor asset condition, however, the coverage of this will belimited to available sensors and areas where the asst may be instrumented, therefore, only data from a specific number and type of parameter are collected. As a result, the picture that the prognostic tool provides is not total. To achieve a more holistic picture of asset and system condition, careful planning and design must take place to integrate RCM, physical monitoring as well as decision makers' assessment is essential. The two key maintenance activities (on-site and off-site condition monitoring) need to be integrated to ensure important data is not lost in the interface between them.

As can be seen, and noted throughout the following subsections, the matter of introducing a new piece of technology within an existing organisational structure does not reside exclusively within the engineering nor the human/social domain. As Lee 16 highlights, when technological systems (the RCM) and social systems (in this case, an organisation) interact, a phenomena emerges to which disciplines from behavioural and technical science both contribute to. A specific field concerned with extended research in this area is the Information Systems field. For example, Larsen and Myers ${ }^{17}$ investigated the success of a business process re-engineering project that involved the implementation of a software-based resource planning system in an organisation, and found that, although the project was catalogued as a success, there were worrying longer term implications for the organisation. Loss of in house expertise through de-skilling of the workforce was a major effect highlighted. Other examples of research 
that look at the impact of technology are included by Hutchins ${ }^{18 ; 19}$ and Walker et al. ${ }^{20}$, which look at the impact of technological systems on situational awareness in the aviation domain. Specifically, these examples investigate the impact of various systems in the cockpit (i.e. information systems) and pilot situational awareness, the question being if the technological systems support (i.e. cognitive efficiency realised through distributed cognition) or limit the pilot situational awareness. The answer is not that straight forward and it is arguable this is an area that continuously preoccupies both technological and human scientists alike. As the RCM is ultimately an information system design to support/ enhance extant decision-making capability, these examples, though not exhaustive, highlight areas of concern when developing and integrating such a system. However, the work reported here is mainly concerned with describing and exemplifying methods that can be used to identify specific organisational challenges (i.e. organising activities, processes, roles and responsibilities) rather than to exhaust all the challenges (technological and human) related to integration of technological systems in organisations.

In the following sections, the paper describes the approach adopted to identify those organisational challenges specific to the integration of the prognostic tool developed by the HPA project within the London Underground maintenance processes. It considers the facilitation of change from reactive to predictive maintenance and increasing likelihood of successful up-take and implementation of the tool and associated processes and discusses the implications of the findings.

\section{Methodology}

\section{Approach}

In order to investigate the pertinent organisational challenges within this project, a three-step approach was implemented $^{21}$.

- 'As is' model - creation of process maps which reflect maintenance processes as they are in practice, or work as done (as opposed to work as planned);

- New system functionality - identification of the functionality to be provided by the new prognostic tool;

- 'To be' model - integration of the additional functionality provided by the new tool to create new process maps for implementation and comparison to 'as is' models to support identification of organisational differences between the existing and future maintenance processes.

\section{Data Collection}

In order to create the relevant process maps, data was collected through a series of workshops with a reflective sample of key stakeholder groups from London Underground, including maintenance delivery, planning and condition monitoring experts. A thorough review of formal documentation (e.g. London Underground work instructions) was also carried out to understand work as planned. A total of 11 maintenance experts (Inspectors, Condition Monitoring Engineers, Planning Engineers, Asset Engineers, Technical Engineers and Performance Managers) have been collectively interviewed during a series of four workshops. These followed a collective semi-structured interview format, with a series of questions and points given to attendees for discussion. Output reports were compiled and shared with London Underground maintenance experts to validate the findings and to facilitate further discussions where necessary.

Furthermore, 17 formal documents including working instructions relating to Plan Preventative Maintenance, Work Management, Inspections, Condition Monitoring and Standards have been reviewed. The data extracted from the documents was used in the creation of maintenance process maps used in workshops as a starting point. The data regarding the prognostic tool functionality was gathered through discussions with the tool developers (our project partner Humaware)

\section{Data and Process Analysis}

In order to analyse the data collected through the workshops, a numder of tools and techniques were used, including:

- IDEF0,

- Process flow diagrams,

- The Role Matrix Technique(RMT).

The first two of these tools allow us to map and model different perspectives on the relevant processes. They are both widely used, IDEF0 for the analysis and modelling of functional aspects of organisations, typically at a strategic level, while the process flow diagrams allow for representation of the flow of information within (and sometimes between) systems, and to capture different types of relationships (e.g. time dependencies) between functions. The combination of these two methods has proven useful for investigating the functional aspects of the maintenance process. 


\section{The Role Matrix Technique (RMT)}

The RMT ${ }^{22}$ was developed to map roles to various stages within a process and to identify the relationship between these roles. It has been successfully used both as a research tool and within a variety of organisations (SMEs to large multinationals). The RMT includes the following steps:

- Description of roles involved in an existing mapped process;

- Decomposition of the process into lower level activities and sub-activities (if this has not been completed previously);

- Allocation of roles to the various activities and subactivities within the process;

- Translation of the role detail onto a two-dimensional matrix top help understand the discretion that the different roles have in achieving their goal and their degree of freedom (Figure 2).

The purpose of employing the RMT is to define and visualise the relationships between the roles in terms of role boundaries, interactions, responsibility and accountability in a given organisational process ${ }^{22}$. In this case the RMT was used not only as a role analysis method ${ }^{7}$ but also as a means to promote discussions between the various London Underground maintenance experts regarding future process configuration and role allocation. Developing multiple versions of the Role Matrix and role allocation also allows for comparison, pre and post change (such as the change in maintenance practice considered here).

As mentioned above, the first step of the RMT process, involves identification and definition of roles. The various roles involved in a process can be performed not only by a human but also by a group (e.g. a team such as 'fault review' team that has to decide on actions following review of asset faults) or an intelligent agent (e.g. a decision-making tool). Furthermore, one agent can have allocated more than one role within a process ${ }^{24}$.

When allocating the roles to the activities within the process (third step in the RMT analysis) four different types of role responsibility have been defined ${ }^{21}$ :

- Controller

- Executor

- Constraining Advisor (gives advice that is unlikely to be ignored)

- Discretionary Advisor (gives advice that can be disregarded)

The identification of the various types of responsibilities that roles have in a given process is necessary as the allocation of the roles to process activities depends on the type of responsibility that a role has ${ }^{23 ; 24}$. Following, the roles within that activity are allocated with the help of the digram depicted in Figure 3. (The numbers in the boxes here simply represent the freest to the most constrained roles).

The last step in the RMT consist of aggregating all of the activity diagrams to create an overall map for the process under investigation and the transfer of the roles onto the Role Matrix (Figure 2). The way in which the roles are plotted onto the matrix depends on the relationships that one role has to another ${ }^{24}$.

There is a certain degree of subjectivity when aggregating the diagrams and plotting the roles onto the Role Matrix. To limit the subjectivity and make the analysis more rigorous one has to follow the rule that there can be only one role in control at any given stage in the aggregation process and if there are two roles in control then the role that is in control for the majority of time and for the critical activities then that role has to be put in the overall stage control ${ }^{23}$. Furthermore, the analysts must have a good understanding of the roles involved in the process ${ }^{21}$.

\section{Findings}

During the initial stages of the research, formal documentary analysis was carried out, alongside discussions with London Underground maintenance experts. Analysing the data gathered through both these avenues revealed inconsistencies between documentation of planned procedures and practices and individual accounts of work practice. Investigating these observed inconsistencies revealed that some of the documents were either no longer in use or not currently updated following ongoing organisational changes in London Underground, which has implications for the processes and/or roles involved. As a consequence, further analysis relied on subject matter expert accounts rather than documentary analysis. Whilst this presented a challenge for data collection, it also indicated the first of our findings.

Following on from this initial data collection and analysis, an 'as is' model was created to reflect the existing maintenance processes. A top level overview of this is presented in Figure 4. In this model, all CM activities, including RCM, are located within the 'Deliver Maintenance' activity.

Analysis also indicates that although basic CM procedures are in place within the overall existing process, the current strategy focuses on reaction to faults and fault preventative practices (e.g. scheduled maintenance based on asset failure history). Furthermore, it was noted that the location of $\mathrm{CM}$ 


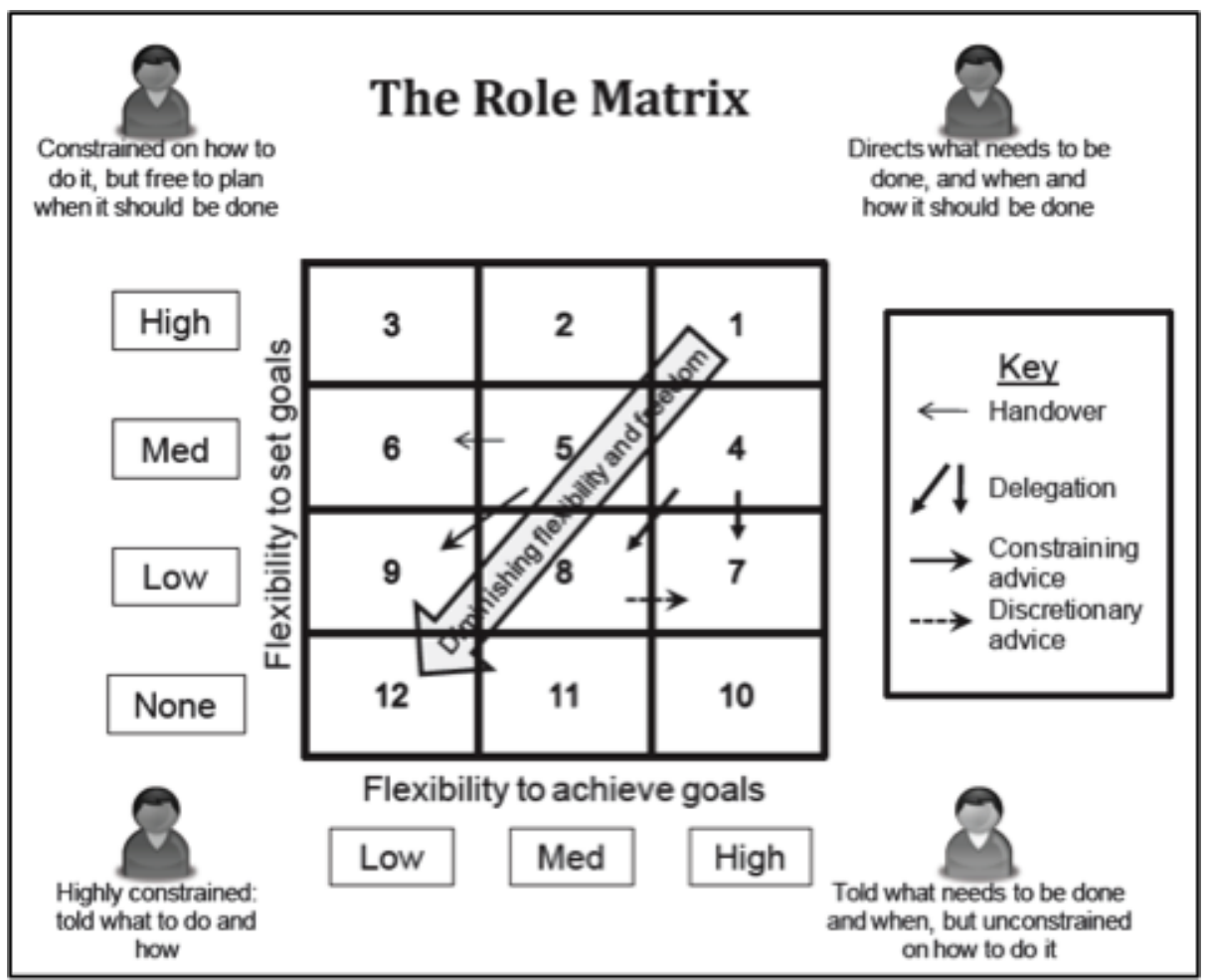

Figure 2. The Role Matrix ${ }^{23}$

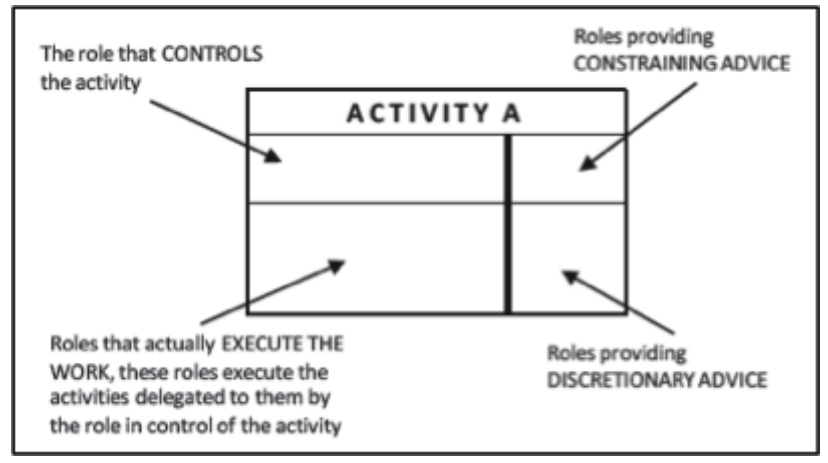

Figure 3. Role analysis representation ${ }^{24}$

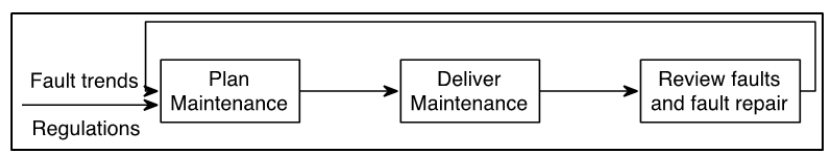

Figure 4. 'As is' Maintenance processes

and RCM activities within the overall maintenance process has implications for how the CM output is used.

For example, the 'Deliver Maintenance' part of the overall Maintenance Process relies on outputs from the 'Plan Maintenance', 'Review faults' and 'Fault repair' processes. However, $\mathrm{CM}$ and RCM information (e.g. Condition Monitoring Reports) is not included formally within the Review process. The outputs from CM Reports seem to be included in the fault review process only when there is a high possibility of asset failure.
Within the maintenance process eight basic roles were identified as having particular importance in the decisionmaking activities. These are described in Table 1.

Following the identification and definition of roles, the Maintenance Process was decomposed in activities and subactivities. Each underlying activity was then analysed (Figure 3) from the point of view of the type of involvement that a role has within that activity. As mentioned above, the type of involvement was based on four identified types of responsibility: controller, executer, constraining advisor and discretionary advisor (following the RMT process). The results where then aggregated, level by level (sub-activities in activities and so on up to the process level) to obtain an overall image of the involvement of the roles in the Maintenance Process. The roles are then positioned on the Role Matrix (Figure 2) to obtain a map of the relationships between these roles. This map is presented in Figure 5.

The matrix in Figure 5 describes, in a graphical form, the position of every role relative to the other roles placed on the grid and relative to the dimensions of the grid. The relationships between the roles are identified through a 'paste function' ${ }^{23}$. For the analysis of the relationships between the roles within the Maintenance Process, four functions have been identified ${ }^{7}$ :

- Delegate 
Table 1. Role Definition - Maintenance Process ${ }^{7}$

\begin{tabular}{|c|c|c|}
\hline Role & Acronym & Definition \\
\hline Asset Group & 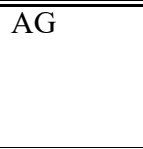 & $\begin{array}{l}\text { The asset group is composed of managers involved in the maintenance process; } \\
\text { e.g. Asset Manager, Technical Manager, Planning Manager. The asset group } \\
\text { decides what and when the 'scheduled' interventions should take place; i.e. } \\
\text { perform strategic planning. }\end{array}$ \\
\hline Planning Team & PT & $\begin{array}{l}\text { Plan the delivery of maintenance interventions; i.e. allocation of maintenance } \\
\text { works, generate work orders and job packaging. }\end{array}$ \\
\hline Duty Shift Manager & DSM & $\begin{array}{l}\text { Oversees day-to-day delivery of maintenance works; i.e. supervise maintenance } \\
\text { interventions, updates MCC, closes work orders, reports faults identified during } \\
\text { maintenance interventions. }\end{array}$ \\
\hline Maintenance Team & MT & $\begin{array}{l}\text { Carry out the maintenance works. Issue reports following the intervention. } \\
\text { Report to DSM. }\end{array}$ \\
\hline Maintenance Control Centre Engineer & $\mathrm{MCC}$ & Handles incidents and fault calls and issues service requests. \\
\hline Daily Failure Meeting Team & DFMT & Review past $24 \mathrm{~h}$ fault reports and incidents. \\
\hline FRACAS Team & FT & $\begin{array}{l}\text { Review past week fault reports and service disruption incidents. Establish route } \\
\text { cause, take appropriate actions such as request monitoring and/or repair work } \\
\text { interventions. }\end{array}$ \\
\hline Condition Monitoring Engineer & CME & $\begin{array}{l}\text { Analyses the condition monitoring data (on-site and remote) and issues } \\
\text { Condition Monitoring Reports. }\end{array}$ \\
\hline
\end{tabular}

- Offer constraining advice

- Offer discretionary advice

- Handover

Regarding the relationship between the various roles involved in the Maintenance process, the findings show that the Planning Team (PT) can ignore the information provided by the Condition Monitoring Engineer (CME) and that the PT executes the intervention plan rather than controlling it. Furthermore, there seems to be no direct path of information flow (or decision-making chain) between condition monitoring (CME) and the planning of the maintenance interventions.

To progress the second stage of the research, discussions were held with the tool developers to determine planned tool functionality. Based on this functionality, a new process was proposed, incorporating it into the existing process, and was detailed in a new 'prognostic maintenance' process map. A top level representative view of this process is included in Figure 6.

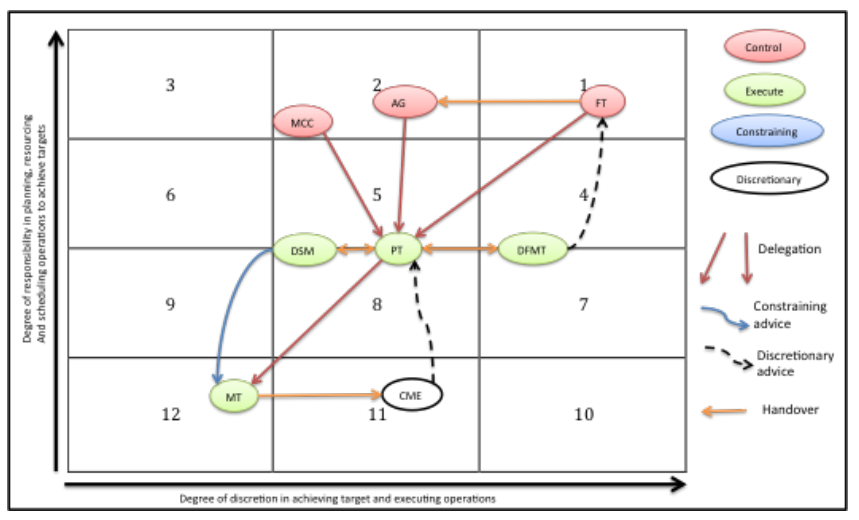

Figure 5. Roles relationships - 'As is' Maintenance Process ${ }^{7}$

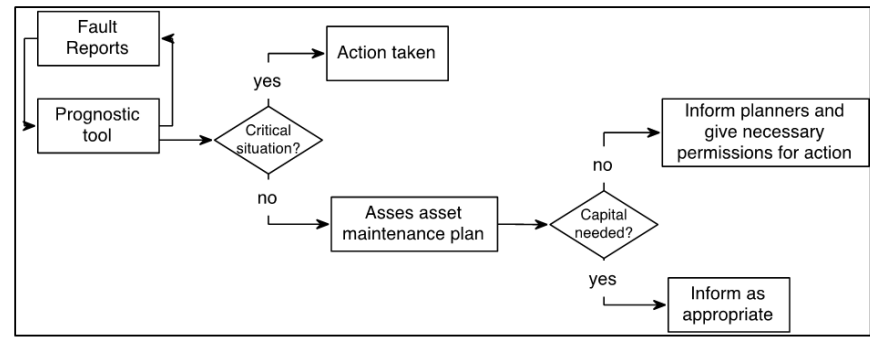

Figure 6. Prognostic Maintenance process

This new process map was utilised, along with the 'As is' model, to lead discussions in a workshop, bringing together London Underground maintenance experts. The workshop investigated the placement of the tool within the organisation and the effect that this may have on roles, responsibilities and existing processes.

As a direct consequence of the introduction of the prognostic tool and because of its capability (issues prognostic RUL and suggests optimum intervention time) a new role has been identified and introduced in the analysis. This is the tool itself (Prg.T) because of its decision- making capability. Furthermore, changes in the attributes of FRACAS Team and CME have been identified as well.

For example, it was established that the FRACAS Team could decide, based on Prg.T output, to modify prescheduled maintenance interventions (change a specific component to eliminate a failure down the line or cancel an intervention that it is not necessary). Furthermore the CME role would have added responsibility, such as, to include Prg.T output in CM analysis to deliver the CM Reports. ${ }^{7}$

This workshop allowed further investigation of how the new technology may integrate and how processes and activities should be organised. Through this, it also revealed 
further insight into existing processes and activities, which had not been evident previously. The findings showed that some activities migrate from their original or planned locations towards others. For example, some of the activities related to 'Plan Maintenance' (Figure 4) were heavily influenced by the 'Delivery' part of the Maintenance Process although according to the process flow diagram it should be the other way around. Initial understanding is that this observed phenomenon might be caused by geographical location of teams involved, although further research would be required to confirm this.

Findings concerning visibility and decision-making power, and how they link, were also determined. For example, the 'Review' part of the process has decisionmaking power over 'Planning Maintenance' however, these processes focus purely on reviewing faults and fault repairs in the existing process (Figure 4), and as such they have limited visibility of works carried out on the asset. In contrast, 'Deliver Maintenance' has full visibility of works, but limited decision-making power over maintenance intervention.

In hindsight of the changes in roles and their responsibilities ('To be' Maintenance Process), a role analysis was performed to highlight these changes. The output of this analysis (The Role Matrix for the 'To be' Maintenance Process) is presented in Figure 7.

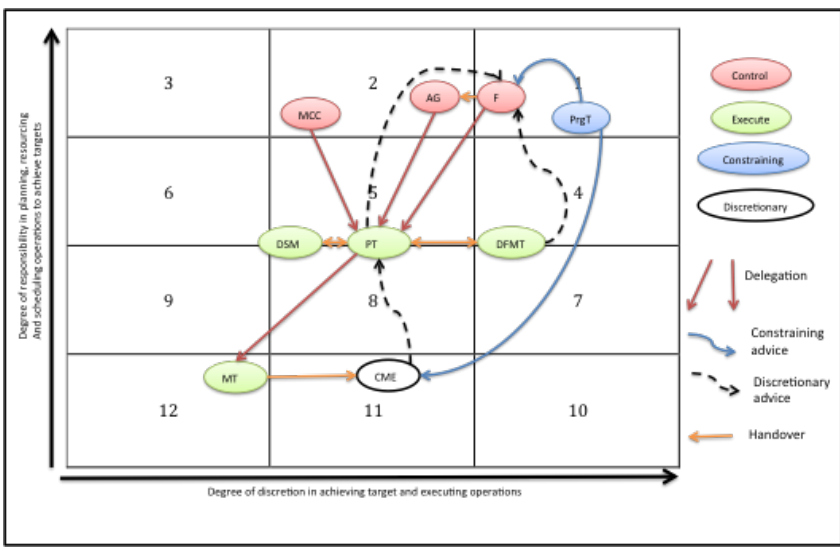

Figure 7. Roles relationships - 'To be' Maintenance Process ${ }^{7}$

Figure 7 shows the immediate impact that Prg.T. will have (given current capability) and how this will influence the $\mathrm{CME}, \mathrm{PT}$ and $\mathrm{F}$ roles. $\mathrm{CME}$ will receive 'constraining advice' (not to be ignored) from the Prg.T. The PT is in a better position to inform $\mathrm{F}$ and Prg.T. can provide valuable advice to F. As a consequence, the FRACAS Team will have to take on more responsibility. For example, when analysing faults and failures the team must take into consideration the $\mathrm{CM}$ Report before requesting changes in the maintenance plan interventions. ${ }^{7}$
However, what the findings reveal following the RMT that is perhaps surprising is that the main planning activities are not influenced strongly by the Prg.T output. That is because of the technical limitations of the tool and the technical limitations of the systems within which it operates, as well as due to the relationship between the tool and its users.

As the current RCM system does not monitor all assets (escalators) one cannot take decisions that will affect the entire fleet of escalators based on condition information related only with a sub-set of the assets. Furthermore, the Prognostic Tool output is based, at the moment, on a limited number of variables thus affecting the quality of the output. However, as more variables are added and the tool is integrated with existing information systems (e.g. Planning

Management System) the Prognostic Tool could have a larger impact in determining the maintenance interventions.

As the Prg.T. will evolve (further extend the RCM capability) and its outputs get more trusted/ valid, the

'advice' provided by CME could have more weight, for example, it could provide 'constraining advice'. That means that CME could potentially move above the PT in the decision making chain.

\section{Discussions and conclusions}

The findings highlight, first and foremost, the importance of having up to date formal documentation regarding the roles and activities within the maintenance process before a technological or structural change is implemented. The documents serve not only as a point of reference for review but are also important in ensuring that the people involved in a process have a clear view of their roles and responsibilities as stated in these formal documents. Lack of or unclear documentation can lead to issues such as: shifting of blame and responsibility, loss of accountability, loss of transparency. Likewise where documentation of processes is not clear this can result in poor understanding of the boundaries of ones own roles and that of other roles, poor communication and data sharing links and problems with delineation of responsibility for decision-making ${ }^{9}$. This does not mean that local tailoring of processes should not take place, but rather that any tailoring should start from a clear recommended baseline and should have a clear and understood rationale.

It is also worth noting that the position of a sub-process or a set of activities within a process can affect the way the output of those activities is utilised. For example, the $\mathrm{CM}$ and $\mathrm{RCM}$ activities, from a process point of view, are currently located within the Delivery part of the Maintenance 
Process. According to this configuration, it is difficult for the $\mathrm{CM}$ and RCM output to have the desired impact on the maintenance regime, because, the Delivery part of the Maintenance Process does not have the necessary decisional power to act upon the knowledge provided by $\mathrm{CM}$ and RCM.

As the prognostic tool is to be integrated with the current RCM system, the positioning of the RCM and CM is crucial if the organisation is to benefit from the enhanced capability provided by the tool. Outputs of the new, enhanced RCM system have not only to be reviewed by people with decisionmaking power to act on the tool output, but the processes through which this is achieved and the relevant connecting links have to be put in place by theorganisation.

Furthermore, re-engineering of the maintenance processes to benefit from the new technology has to be complemented by decisions regarding geographical location and means of interactions between the roles and the departments. As findings have indicated, in some situations, individual alliances towards certain groups can have an effect on the activities carried out. The effect is not necessarily negative, and in some instances it is actually desirable as it might bring huge benefits, but it needs to be understood if it is to be exploited. As mentioned in the first part of the paper, this effect may appear also as a result of human optimisation of their work and environment; effect that is highlight also by Jonsson and colleagues ${ }^{4}$ in their study on exploring the challenges of integrating IT-based services for remote diagnostics. The point is though that this effect has to be acknowledged and understood as this has an effect on the way the activities within a process are actually carried out.

Having a structured, sound process, which has been resourced with clear roles (each of which will have a role profile) and consensus on, and an understanding of other factors that can affect the running of the process before a change is implemented, can bring huge benefits. Investing in understanding and creating this partial enterprise model and associated process configuration which is suitable for a specific change within a specific organisation could bring that competitive advantage that technology on its own cannot achieve.

\section{Funding}

This research is part of the 'Health and Prognostic Assessment of Railway Assets for Predictive Maintenance project', which is part of the 'Enabling the Digital Rail' research programme, funded jointly by Innovate UK and the Rail Safety and Standards Boards (RSSB).

\section{Acknowledgements}

Along with the funders, the authors would also like to acknowledge London Underground and other partners in the project: Telent, Humaware and University of Nottingham, for the support provided. The views expressed in this paper are those of the authors and do not necessarily represent those of the other project partners or funding bodies.

\section{References}

1. Iung $B$, Veron $M$ and Suhner $A M$ ans Muller. Integration of Maintenance Strategies into Prognosis Process to DecisionMaking Aid on System Operation. In CIRP AnnalsManufacturing Technology, 1, volume 54. pp. 5-8.

2. Al-Najjar B and Alsyouf I. "selecting the most efficient maintenance approach using fuzzy multiple criteria decision making”. Int Journal of Production Economics 2003; 84: 85100.

3. Marais KB and Saleh JH. Beyond its Costs, the Value of Maintenance: An Analytical Framework for Capturing its Net Present Value. In Reliability Engineering and System Safety, 2, volume 94. pp. 644-657.

4. Jonsson K, Holmstrom $\mathrm{J}$ and Leven P. Organisational dimensions of e-maintenance: a multi-contextual perspective. International Journal of System Assurance Engineering and Management 2010; 1(3): 210-218.

5. Kajko-Mattsson M, Karim R and Mirjamsdotter A. Fundamentals of the eMaintenance Concept. In 1st International Workshop and Congress on eMaintenance. Sweden.

6. Karim R and Soderholm P. Application of information and communication technology for maintenance support information services. In Journal of Qualitative Maintenance Engineering, 1, volume 15. pp. 78-91.

7. Ciocoiu L, Siemieniuch C and Hubbard EM. Using the Role Matrix Technique to Identify Organisational Changes: A Practical Study within London Underground Escalators Maintenance Service. In IEEE ISSE.

8. Mobley R. An Introduction to Predictive Maintenance (2nd Edition). Butterworth-Heinemann, 2002.

9. Koochaki J and Bouwhuis IM. The role of knowledge sharing and transactive memory system on condition based maintenance policy. In IEEE International Conference on Industrial Engineering and Engineering Management, Singapore.

10. Hodgson EM Aand Hubbard and Siemieniuch CE. Toward an understanding of culture and the performance of teams in complex systems. IEEE Systems Journal 2013; 7(4): 606-615.

11. Hodgson A, Siemieniuch C and Hubbard EM. Culture and the safety of complex automated sociotechnical systems. IEEE 
Transactions of Human Machine Systems 2013; 43(6): $\quad 608$

619.

12. Chapanis A. Human factors in systems engineering. Systems Psychology 1970; : 51-78.

13. Hollnagel E. Control versus dependence - striking the balance in function allocation. CSERIAC Gateway 2011; 9: 12-13.

14. Sheridan TB. Human supervisory control. Design of work and development of personnel in advanced manufacturing. John Wiley Sons, 1994.

15. Sheridan TB. Humans and Automation. Systems Design and Research Issues. Human Factors and Ergonomics Society, 2002.

16. Lee A. Editorial. MIS Quarterly 2001; 25(1): iii-iv.

17. Larsen MA and Myers MD. When success turns into failure: a package-driven business process re-engineering project in the financial service industry. Journal of Strategic Information Systems 2000; (8): 395-417.

18. Hutchins E. How a cockpit remembers its speeds. Cognitive Science 1995; (19): 265-288.

19. Hutchins E. Cognition in the Wild. MIT Press, 1995.

20. Walker GH, Stanton NA, Salmon PM et al. How can we support the commander's involvement in the planning process? an exploratory study into remote and co-located command planning. International Journal of Industrial Ergonomics 2009; 39(2): 456-464.

21. Ciocoiu L, Hubbard EM and Siemieniuch C. Implementation of remote condition monitoring system for predictive maintenance: An organisational challenge. In Contemporary Ergonomics and Human Factors, Proceedings of. Taylor and Francis.

22. Siemieniuch CE and Sinclair MA. On complexity, process ownership and organisational learning in manufacturing organisations, from and ergonomist perspective. Applied Ergonomics 2002; 33.

23. Callan K, Siemieniuch C and Sinclair M. A Case Study Example of the Role Matrix Technique. International Journal of Project Management 2006; : 506-515.

24. Henshaw M, Maged M, Siemieniuch CE et al. Identification of induced complexity in product service system enterprises. Journal of Enterprise Transformation 2011; 4: 269-289. 